\title{
Quelques réflexions sur les pertes de charge
}

\section{Some reflections on head losses}

\author{
PAR L. YADO'T
}

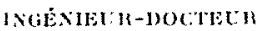

\begin{abstract}
Rappel de lietul actuel - et classique - de ha question des pertes de charge. ... Examen detaillé de linfluence de deux elements fondamentaur: nature de la paroi et forme de la section. - L'importance el l'intéret des travaux de Vikuradse, le parti qu'il convient d'en tirer. - Notion de « densife de rugosité $\gg ;$ transition entre le tnrbulent lisse et le turbnlent rugneux. - Trawaux de Colebrook; proposition d'une formule de perte de rharge couvrant à la fois le turbulent lisse ef le turbulent rugueur, et respectant les particularités du régime Iransitoire. - Critique de la notion de rayon hydralique (sections de forme tourmentee; répartition non uniforme de rugosité; les pertes de charge partielles ef la loi de Dalton). libre.
\end{abstract}

\begin{abstract}
Reference to present and rlassic slate of the ruestion of head losses. .... Detaled axaminafion of the infuence of two fundamental elements: nature of the wall and shape of the section. - The importance and interest of the Nikuradse works and what should be learned from them. - Ideat of "density of roughness;" transition between fully turbalent flow and smooth boundary turbulent flow. The works of Colebrook; proposition for a head loss for mula covering both fully and smooth boundary turbulent flows and respecting the charteteristir's of the transition regime. Criticism of the hydratic radius idea (distorted sections; non uniform distribution of roughness; partial head losses and the Dalton law). - Remarks on free surface eanals.
\end{abstract}

Il n'y a pas de probleme de mécanique des fluides qui ait donne naissance a un plus grand nombre de formules que celui des pertes de charge. La multiplicité des recettes relatives a ce sujet est une preuve de l'importance de la question, mais aussi, malheureusement, une cause de perplexité pour celui qui doit les utiliser. Cet état de chose est dû à plusieurs raisons :

$1^{\circ}$ Le problème des pertes de charge fait intervenir de nombreux parametres, dont il n'esi pas toujours facile de tenir compte et que beaucoup d'auteurs ont négligé ou ignoré en partie;

2 "Les résultats d'une expérience faite sur un type de canalisation ne sont pas toujours directement transposables à une autre canalisation;

3" La complexite des formes de canaux et la diversité des natures de paroi ne permettent pas toujours de se ramener à des schémas simples de calcul;
4" L'étude théorique du problime des pertes de charge est étroitenent liée à l'étude de deux autres problèmes (turbulence et couche limito) qui ne sont apparus qu'asse\% récemment dans l'hydratidgue et dont on ne saurait dire encore mantenant qu'ils sont completement écaircis.

A l'heure actuelle, il n'existe pas de théoric vaiment lumineuse et complete de la question dis pertes de charge, mais plutôt un assemblage de résultats basés sur l'expérience et plus ou moins étayés par des raisonnements empruntés a la theorie de la turbulence of de la couche limite. Némmoins, le plus grand pas a éle fivi puisque lon a dénombré les facteurs essentiels du problème et déterminé, par l'expérience, le sens de leur influence. Ces facteurs fondamenlaux sont de deux sortes : les uns de nature géometrique (dimensions ef forme de la section, forme de la paroi), les autres de nalure hydraulique (viscosité, nombre de Rernolos). 
Nous nous proposons loul d'abord de rappeler à grands traits l'essentiel des connaissances classiques de la question, ne serait-ce que pour mieux situer les quelques points particuliers que nous examinerons plus en détail.
Limitons loul d'abord notre domaine. II ne sera question ici que des pertes de charge en conduite rectiligne à section constante en régime établi et permanent, pour un fluide incompressible.

\section{I. - RAPPEL DE NOTIONS CLASSIQUES}

\section{Force TANGeNTHELLE A LA PAROI ET RAYON HYDRAULIQUE}

Découpons dans la veine liquide d'une conduite le prisme liquide compris entre deux seclions droites séparées par la distance $>l$. Soit $\omega$ la surface de la section droite, $P$ son périmètre, $p+\lrcorner p$ la pression dans le plan de la première section et $p$ la pression dans le plan de la

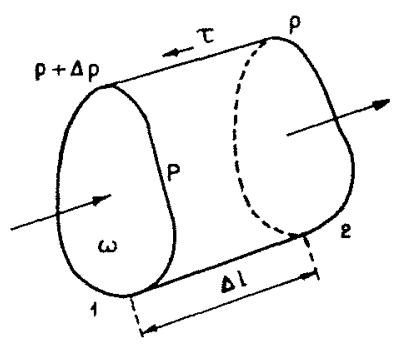

Frg. 1

deuxième section. Soit enfin $\tau$ la force tangentielle créée en un point de la paroi par le frottement liquide. Ecrivons l'équilibre suivant l'axe de la conduite, du prisme liquide considéré sous l'action des forces de pression et des forces tansentielles. On a :

$\iint_{\omega 1}^{\infty}(p+\Delta p) d \omega-\iint_{\omega_{2}}^{\infty} p \cdot d \omega=\Delta l \cdot \int_{\mathrm{p}}^{\infty} \tau d \mathrm{P}$

La pression étant supposée constante dans une unême section, on aura plus simplement :

$$
\text { (1) } \Delta p=\perp l \cdot \int_{\mathrm{P}} \tau d \mathrm{P}
$$

La perte de charge apparaît alors :

$$
\frac{\Delta p}{\Delta l}=\frac{1}{\omega} \int_{\mathrm{P}} \tau d \mathrm{P}
$$

Admettons alors que la force tangentielle : ait la même valeur en tout point du périmètre de la section. On obtient alors la formule classique de perte de charge:

$$
\frac{\Delta P}{\Delta l}=\tau \cdot \frac{P}{\omega}
$$

Pour une section circulaire, ceci s'écrit :

$$
\frac{\Delta p}{\Delta l}=-\frac{2}{\mathrm{R}}
$$

$R$ étant le rayon de la section. On donne généralement au rapport $\omega / \mathrm{P}$ le nom de rayon hydraulique, que l'on écrit $\mathrm{R} h$, d'où :

$$
\frac{\Delta p}{\Delta l}=\frac{\tau}{\mathrm{R} h}
$$

L'apparition du rayon hydraulique permet d'escamoter la forme de la section et d'appliquer la formule précédente à un canal de section quelconque. Il est bon, tontefois, de ne pas oublier que ce tour de passe-passe n'a de valeur que dans la mesure où l'hypothèse de la constance de l'effort tangentiel $\tau$ le long du périmètre de la section est bien vérifiée. En toute rigueur, cette hypothèse n'est vérifiée que dans deux cas : le canal à section circulaire et le canal compris entre deux plans parallèles indéfinis. Nous verrons par la suite ce qu'il faut penser de l'influence de la forme de la section du canal et considérons, pour le moment, l'hypothèse de la constance de $\tau$ comme valable.

\section{Forme habrtuelle de l'Expression DES PERTES DF CHARGF}

L'expérience ayant montré que, dans la plupart des cas, les forces tangentielles $\tau$, c'est-àdire en définitive les pertes de charge, étaient sensiblement proportionnelles au carré des vitesses d'écoulement, on adople une expression mettant ce fait en évidence. Soit :

$$
\frac{\Delta p}{\Delta l}=\lambda \frac{\rho u^{2}}{2 \mathrm{D}}
$$

expression dans lacuclle $\lambda$ est une constante sans dimension, ou coefficient de perte de charge, ? la masse spécifique du fluide, $"$ la vitesse 
moyenne d'écoulement dans la conduite et D le diametre de celle-ci (dans le cas d'une conduite non circulaire, D sera remplacé par $4 \mathrm{R} h$ ). On va voir tout de suite d'où vient le 2 du dénominateur; exprimons, en effet, la perte de charge en hauteur de fluide, ce qui revient à diviser le

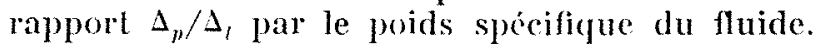

On aura :

$$
\frac{\Delta h}{\Delta l}=\frac{1}{\gamma} \frac{\Delta p}{\Delta l}=\frac{\lambda}{\mathrm{D}} \frac{u^{2}}{2 g}
$$

Le coefficient $\lambda$ ainsi défini suffit à caractériser une perte de charge. Pour une conduite et un type de paroi donné, le coefficient $\lambda$ varie avec

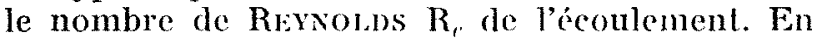
général, trois régimes sont à considérer suivant la valeur du nombre de Rexions : aux très faibles valeurs de $R_{r}$ jusqu'à 2.000 environ le régime laminaire; au delà de 2.000 , le régime turbulent qui, lui-même, comprend aux basses valeurs de $R_{t}$ le régime turbulent lisse, et aux grandes valeurs de $\mathrm{R}_{c}$. le regime turbulent rugueux. La transition entre ees deux régimes s'étale sur une bande ítendue de valeurs de $\mathbf{R}_{c}$, valeurs dépendant d'ailleurs de la rugosité relative de la paroi.

\section{RÉgIME LAMINAIRE}

Le régime laminaire, d'un intérêt industriel restreint, est le seul qui soit vraiment accessible au calcul : les forces de viscosité étant seules à intervenir et la nature de la paroi n'ayant pas d'influence. Pour une conduite à section circulaire, le coefficient de perte de charge à la valeur :

$$
\lambda=\frac{16}{\mathbf{R}_{f}} \quad \text { avec } \mathbf{R}_{f}=\frac{\mathrm{D} u}{y}
$$

pour un écoulement entre deux plans paralliles distant de $h$, on a :

$$
\lambda=\frac{48}{\mathrm{R}_{r}} \quad \text { avec } \mathrm{R}_{\rho}=\frac{h u}{\nu}
$$

pour un écoulement dans un canal à section carréc de côté $a$, on $\mathbf{a}$ :

$$
\lambda=\frac{14,2}{\mathrm{R}_{e}} \quad \text { avec } \mathrm{R}_{\varepsilon}=\frac{a n}{\nu}
$$

Nous donnons ces quelques chiffres pour bien indiquer que, en régime laminaire, la perte de charge n'est pas indépendante de la forme de Ia section.

Notons, à ce propos, une curieuse particularilé des icoulements laminares. On réprésente habituellement la répartition des vitesses dans une section par un ensemble de courbes isotaches. Or, dans le cas d'un écoulement laminare, ces courhes isotaches sont on même temps les lignes de courant d'un champ rotationnel à densité tourbillonnaire constante limité an contour de la section. Considérons, en offel, une zone de la section comprise entre deux courbes telles que

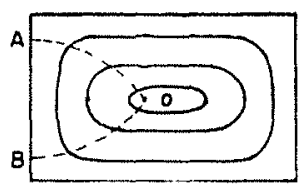

Fra. 2

AO et BO orthogonales atux courle's isotaches. Ecrivons l'équilibre de l'élément fluide correspondant à cette zone sous l'action de l'ensemble des forces de pression et des forces tangentiolles de viscosité. Soit $S$ la surface de la zone considéré, $\Delta p$ le gradient de pression suivant laxe de la conduile, i la force tangenticlle ef $\Delta s$ le segment de la paroi qui limite la zone considérée. On a $\lrcorner p . S=\tau\lrcorner$.$s . La mème relation$ s'applique si, au lieu de limiler la zone à la paroi, on la limite à une isotache queleonque; mais, de plus, la force tangentielle a pour expression : $\tau=\mu(d n / d n), d v / d n$ étant le gradient de vitesse compté normalement aux isotaches. Considerons un accroissement fini de vitesse $\Delta z$ correspondant a un espacement $\Delta n$ des isotaches; on aura encore $\tau=\mu(\Delta v / \Delta n)$; par suite, en reportant cette valeur de $\tau$ dans la premiere expression, il vient :

$$
\frac{\Delta s}{\Delta n}=\frac{\Delta p \cdot S}{\mu \Delta v}=C^{\prime n} \mathrm{~S}
$$

La relation qui lie les côlés $\Delta_{*}$ et $\Delta_{n}$ des quadrilalères élémentaires du réseau des isotaches et de leurs orthogonales est bien celle que doivent vérifier les champs rotationnels a densité tourbillonnaire constante. Par suite, le faisceau des isotaches représentatif de la répartition de vitesse dans la section d'une conduite en régime laminaire, représente également les lignes de courant d'un champ rotationnel linite par une ligne de courant affectant la forme du contour de la section droite de la conduite.

\section{RÉGIME: TURBULENT}

Passons mantenant au rigime turbulent ef, tout d'abord, au turbulent lisse. Ce regime est caractérisé par le fait que la nature de la paroi est sans influence sur la perte de charge, la hau- 
leur des aspérités restant nettement inférieure a l'épaisseur de la couche laminaire. Le coeflicient $\lambda$ diminue quand le nombre de Revocos augmente suivant ba loi de Pravoru-Kaksax :

$$
\frac{1}{\sqrt{r}}=2 \log \frac{\mathrm{R} \cdot \sqrt{\pi}}{2,51}
$$

expression qui, jusqu’à $R_{i}=10^{\circ}$, peut ètre remplacée par l'ancienne formule plus simple de BuAsILS:

$$
\lambda=0,316 \mathrm{R}_{e^{\prime}}-\cdots, 4
$$

Le régime turbulent rugueux est caractérisé par le fait que les aspérites de la paroi sont completement sorties de la couche laminaire, ce qui conduit à une perte de charge rigoureusement proportionnelle au carré de la vitesse. Pour une conduite et un type de paroi donné, le coefficient $\lambda$ devient done indépendant du nombre de Revionos. Par contre, il se trouve sous la dépendance des caractéristiques géométriques de la rugosite de paroi. Nikuranse a chabli la relation qui lie le coefficient $i$ a la hauteur des aspérités, pour un type donni de rugosité cet non pas pour n'importe quel type de rugosité) :

$$
\lambda=\quad(2 \log \mathrm{R} / \mathrm{K}+1,74)^{2}
$$

ou, ce qui revient au même :

$$
\frac{1}{\sqrt{\lambda}}=2 \log \frac{3,711}{K}
$$

$R$ ćtant lo rayon de la conduite et $K$ le diamètre des grains de sable qui, collés à peu près jointifs à la paroi, constituent les aspérités. On ne saurait trop insister sur cette derniere définition du terme $K$ trop souvent dénommé « hauleur des aspérités ». Etant donné la disposition des grains de sable selon Nukuradse, la hauteur d'aspériti correspondante au sens habituel, e'est-à-dire la distance de creux à plein, est sensiblement égale a la moitié du diamètre des grains de sable. Afin d'éviter toute ambiguïté, lorsqu'il s'agira d'une rugosite type Nikinadse, on affectera la lettre $\mathrm{K}$ d'un indice $\mathrm{N}$, soit $\mathrm{K}_{\mathrm{x}}\left(^{*}\right)$.

(*) Le véritable intérêt des travaux de Nikuradse n'est pas d'avoir douné une nouvelle formule de perte ds charge parmi tant d'autres. mais bien d'avoir systématiquement étudie l'influence de Ja hauteur des aspérités. Il est regrettable que la plupart des manuels d'hydraulique donnent cette formule comme d'un usage courant, sans préciser les conditions très spéciales auxquelles elle s'applique. C'est une erreur fréquente de vouloir lui faire dire ce pour quoi elle n'est pas faite. Son application, par exemple, aux revêtements bétonnés $11^{\prime} a$ pas grand sens, car la nature géométrique de la rugosité type enduit bétonné est fort differente de la nature
L'ensemble des risultats de NikvRadse est généralement représenté sur un diagramme désormais classicfue, comportant un laisceau de courbes avec le rapport $R / K$ comme parametre.

On peut, par une anamorphose, ramener ce faisceau de courbes à une seule courbe. Soil $\sigma_{11}$ l'effort tangentiel moyen a la paroi, la quantité $\sqrt{\frac{\tau_{x}}{o}}$ est homogène à une vitesse; on l'appelle généralement vitesse de friction ef on l'écrit $V_{*}$. On réalisera l'anamorphose dont il esl question,

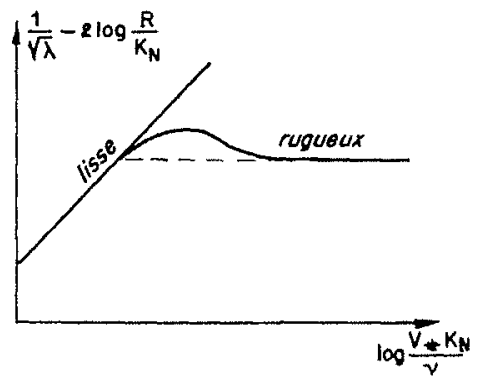

Fic. 3

en étudiant les variations de la quantiti $\frac{1}{\sqrt{\lambda}}-2 \log \frac{R}{K_{Y}}$ en fonction de $\log \frac{V_{*} K_{V}}{y}$.

Le domaine du lurbulent lisse sera représenté par la droite :

$$
\frac{1}{\sqrt{h}}-2 \log \frac{R}{K_{x}}=0,8+2 \log \frac{V_{*} K_{x}}{v}
$$

et le domaine du turbulent rugueux par l'horizontale :

$$
\frac{1}{\sqrt{i}} \cdot 2 \log \frac{\mathrm{R}}{\mathrm{K}}=1,74
$$

Ces deux droiles se raccordent par mo courbe qui représente la zone de Iransition.

Le passage du turbulent lisse au lurbulent rugueux se manifeste également par une modilication de la loi de répartition de vitesse, suivant un diametre d'une section circulaire. Si on appelle " la vitesse en un point situé à une distance $y$ de la paroi, on a en écoulement turbulent lisse :

$$
\frac{u}{V_{*}}=5,5+5,75 \log \frac{y V_{*}}{y}
$$

et en turbulent rugueux :

$$
\frac{u}{V_{*}}=8,48+5,75 \log -\frac{y}{K_{x}}
$$

géométrique de la rugosité Novradse. Nous dirons enfin qu'en toute rigueur son application industrielle est tres limitée car elle correspond à un type de rugosité qui se rencontrc rarement dans la pratique. 
cetle derniere expression correspond à une rugosité type Nikuradse; dans la zone de transition, la loi de répartition de vilesse est plus complexe et dépend en outre du rapport $K / \delta$ de la hauteur des aspérités à l'épaisseur ò de la couche limite laminaire; cette derniere est d'atleurs liec à la perte de charge par :

$$
a=\frac{11,6 y}{v \frac{\tau_{11}}{\rho}}
$$

'TRANSITION ENTRE LE JISSE GT IE RUGUEEX

La zone de transition entre le régince lisso et le régime rugueux revêt, en pratique, unc srande importance, car, d'une part, pour une conduite donnée, elle est assez étendue (pour fixer les idées, elle couvre une zone de loy $R_{\text {, }}$ (lo 1,5 a 2 ), et, d'autre part, elle correspond tres souvent à la gammo des nombres de Riswords les plus utilisés. Ce fait est très certainement la principale raison de la diversité et de l'incohérence de la plupart des formules de pertes de charge. Les études relativement récentes de NikURADse, de Colebrook, entre autres, ont permis de commencer à démêler le problème, mais celui-ci est rendu particulierement ardu par la diversite des types de rugosité que l'on reneontre dans la pratique.

Niktradse obtient expérimentalement, dans la zone de transition, une inflexion caractéristiquo de la courbe représentative des variations de $\lambda$. Cette inflexion n'est pas générale, mais doit être considérée comme liée au type assez particulier de rugosilé étudié par cet auteur.

Colsbrook, par contre, a étudić plusieurs types de rugososités schématiques, constitués par do grosses aspérités réparties sur un fond lisse ou rugueux. Il obtient ainsi des resultats plus roisins de ceux obtenus sur conduites industriclles. que ceux de Nikvadost. Il donne pour la zanc de transition une valeur de ifixée par lat relation:

$$
\frac{1}{\sqrt{\lambda}}=2 \log \left\lfloor\frac{\mathrm{R}_{e} V \lambda}{2,51}+\frac{3,7 \mathrm{~N}}{\mathrm{~K}}\right\rfloor
$$

Nous verrons plus loin ee qu'il faul penser de cette relation qui, d'ailleurs, ne represente que partiellement les phénomènes.

La lransition est essenticllement caractérisé par le fait que, la hauteur des aspérités étant de l'ordre de grandeur de l'épaisseur de la conche laminare, on roit intervenir simultanement les caractéristiques séométriques de la rugosité et le nombre de Rervolos. On a coutume de dire que les aspérités commencent ì émerger de la couche laminaire : ce n'est evidemment que tris grossièrement vai. La couche laminaire n'est pas une couche d'un fluide particulier tapissant simplement la paroi et dont les aspérités sortent a la maniore d'îlots rocheux. En fail, d'une part les asperites reagissent sur la couch laminare bien avanl que d'en émerger (au sens hrodrostatique du mot of d'autre part, le mot laminaire prête à confusion; des observalions microscopiques ont, en effet, montré l'existence dans cette couche, d'imporlants mouvements d'agitation; le mot laminaire s'applique au comportement d'ensemble de la couche, et non au détail de l'écoulement. Quoi qu’il en soil, on peut considerer le régime comme transitoire entro les valeurs suivantes du rapport $\mathrm{K} / \delta$ de la hauteur d'aspérité, à l'épaisseur de la couche laminaire $\mathrm{K} / \hat{s}=0,25$ a $\mathrm{K} / \delta=6$.

Tel est, en gros, l'aspect elassique actuel de la question des pertes de charge en conduite sams surface libre.

\section{II. - INFLUENCE DE LA NATURE DE LA PAROI}

Nous allons nous attacher maintenanl à une étude plus détaillée des caractéristiques de la rugosité, et de leur influence sur la perte de charge. L'état de la paroi demande à étro examiné de deux points de vue dillérents; du point de vue hydraulique et du point de vue gémetrique. Hydrauliquement, on caractérisera l'étal de paroi par la loi de perte de charge. Nous définirons ainsi quatre types de comportement : de paroi :

$1^{\circ}$ Le comportement lisse : qui répond à la loi de Prandtr-Kanmax du régime dit lurbulentlisse. Se rencontre sur paroi lisse ou sur paroi rugueuse, si les asperités sont noyées dans la couche laminaire;

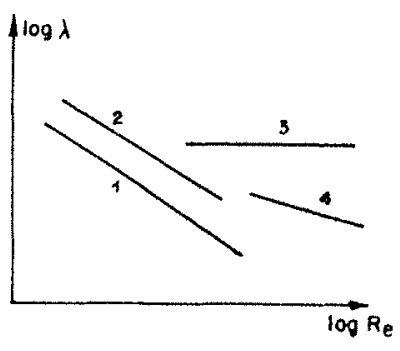

Viri. 4 
$2^{\circ}$ Le comportemenl ondulé : apparenté au lisse el représenté par une loi de variation de $\lambda$ identique à celle du comportement lisse à unc facture près.

$$
\lambda_{\text {ondule }}=\mathrm{C}^{\text {te }} \lambda_{1 \mathrm{ksc}}
$$

se rencontre sur paroi lisse mais ondulée avec ondulation de faible amplitude (par exemple avec certains enduits goudronnés). Tout se passe comme si les ondulations n'apportaient aucune modification au régime turbulent lisse, mais entrainaient seulement un accroissement de la surface des parois.

$3^{\circ}$ Le comportement rugucux : caractérisé par une proportionnalite rigoureuse des pertes de charge au carré des vitesses, c'est-à-dire une indépendance de $\lambda$ vis-à-vis de $R_{6}$.

$4^{\circ}$ Le comportement semi-rugueux : représentant toute la gamme des lois intermédiaires entre le lisse et le rugueux. Se rencontre chaque fois que la paroi comporte, en proportions variables, des plages rugueuses à côté de plages lisses. Ce comportement est industriellement assez fréquent.

\section{PARAMĖTRES CARACTÉRISANT LA RUgOSITÉ}

Du point de vue géométrique, nous envisagerons deux types de rugosité. Tout d'abord, les rugosités à forme d'aspérité et distribution aléatoires dont la rugosité Nikuradse est le type parfait. C'est dans cette catégoric que se rangent la plupart des rugosités industrielles. Puis viennent les rugosités à forme d'aspérité définie et distribution périodique, qui permettent l'étude de l'influence des difficultés caractéristiques et peuvent servir de base de comparaison ou de rugosite étalon.

Jusqu'à présent, c'est la rugosité Nikuradse qui semble avoir jouć ce rôle de rugosité étalon, car les essais de Nikuradse étaient les seuls à présenter un caractère suffisant do rigueur et de généralité. Si la distribution aléatoire des aspérités est, comme nous le verrons, un élément favorable, par contre, le choix du grain de sable (d'un certain grain de sable) comme aspérité est moins heureux, car plus difficile à reproduire avec rigueur. Ce dernier point explique, sans doute, la difficulté que certains expérimentateurs éprouvent à retrouver les résultats de Nikuradse, principalement dans la zone de transition.

Considérons une rugosité artificielle réalisic en disposant, suivant un schéma donné, des aspérités toutes semblables, sur un fond lisse. Cette disposition fait intervenir un certain nombre de paramitres :
1) Forme géométrique de l'aspérilé;

2). Orientation de l'aspérité par rapport à la paroi ;

3) Orientation de l'aspérité par rapport au coutrant;

4) Dimension de l'aspérité (choix d'une dimension caractéristique $K$ de laspérité, par exemple sa hauteur);

5) Forme du dessin d'implantalion des aspórités sur le fond;

(6) Orientation de ce dessin par rapporl at coltrant;

7) Dimension du dessin d'implantation, représentée par une grandeur caractéristique $\mathrm{K}^{\prime}$ de ce dessin, ou micux, par le rapport $\mathrm{K}^{\prime} / \mathrm{K}$.

On voit ainsi la grande diversité des solutions possibles, même en partant d'éléments simples. Si on se propose de réaliser une rugosité artificielle destince à jouer le rôle de rugosité étalon, il y a évidemment intérêt à réduire le nombre des degrés de liberté. On s'efforcera, en pirticulier, d'éliminer les degrés de liberté d'orientation. A ce point de vue, l'adoption d'une aspérité constituée par une sphère serait indiquée si, toutefois, il n'y avait pas lieu de craindre que la diversité de régimes d'écoulement autour d'une sphère ne soit cause de difficulté. Il est au contraire préférable d'adopter des aspérités à arêtes vives pour lesquelles cet inconvénient n'est pas à redouter, cube, pyramide, par exemple. On f'era disparaître l'effet d'orientation en choisissant pour chaque aspérité une orientation au hasard. De même, en dispersant les aspérités au hasard, on fera disparaitre l'effet de l'orientation du schéma d'implantation (c'est ce que fait Nukuranse). Finalement, pour un type d'aspérité donné, il ne subsistera plus que deux paramètres caractéristiques : la hauteur $K$ de l'aspérité et la distance moyenne $\mathrm{K}^{\prime}$ entre deux aspérités ou, ce qui revient au même, le nombre d'aspérités par unité de surface, c'est-à-dire la densité des aspérités.

\section{INFUUNCE DE LA DENSITÉ DES ASPÉRITÉS}

Les travaux de Nikuradse ont montré quelle était l'influence du facteur K. On a vu, en partieulier, que le coefficient $\lambda$ était sous la dépendance du rapport R/K. Examinons maintenant l'influence du facteur $K$, e'est-à-dire du nombre d'aspérités par unité de surface. Les essais de Nikuradse, par suite du mode de réalisation de la rugosité, n'ont pu mettre en évídence l'influence de ce facteur. D'autres anteurs, et principalement Schlichting, ont étudié ce problème. 
Les essais relatifs à cette question ne sont malheureusement pas assez complets, en sorte quil n'est guère possible que d'indiquer l'allure des phénomènes.

La variation du facteur $K^{\prime}$ correspond à la variation du nombre d'aspérilés par unité de surface de la paroi. Pour une aspérité et une conluite données, on peut, pour chaque valeur de $\mathrm{II}^{\prime}$, déterminer une courbe représentative des variations de $\lambda$ en fonction de $\mathrm{R}_{\varepsilon}$. On obtiendra ainsi un faiscean de courbes ayant $K^{\prime}$ pour paramètre. Toutes ees courbes ont une partie commune qui correspond au régime turbulent lisse. Au point $A$, les différentes courbes divergent, la position du point A dépend uniquement de la dimension de l'aspérité par rapport à l'épaisscur du film laminaire. Pour les grands nombres de
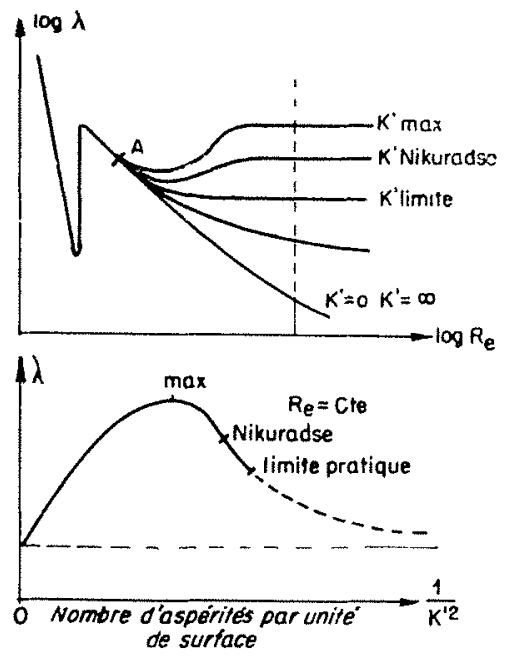

Fig. 5

Reynolds, au-delà de la transition, $\lambda$ est donnćc par une expression de la forme:

$$
\frac{1}{\sqrt{\lambda}}=2 \log \left[(1-\varphi) \frac{\mathrm{R}_{e} \sqrt{\lambda}}{2,51}+\varphi \frac{3,71)}{\mathrm{K}}\right]
$$

expression dans laquelle o est une fonction du nombre d'aspérité par unité de surface, ou fonclion de densité. Cette fonction s'annule pour $\mathrm{K}^{\prime} / \mathrm{K}=\infty$ (pas d'aspérité), et pour $\mathrm{K}^{\prime} / \mathrm{K}=0$ aspérités infiniment rapprochées), clle prend la valeur 1 pour une valeur de $\mathrm{K}^{\prime} / \mathrm{K}$ qui correspond à la disposition de Nikuradse. En dehors de ces quelques points, la fonction o n'est pas connue. Il serait très intéressant de faire des essais systématiques en vue de sa détermination.

Physiquement, on concoit facilement l'aliure de la variation pour les grands nombres de ReYNoLDs de $\lambda$ en fonction de la distance moyenne $\mathrm{K}^{\prime}$ qui sépare deux aspérités. Lorsque $\mathrm{K}^{\prime}$ est infini, les aspérités sont infiniment espacées et on retombe sur le cas d'une paroi lisse; $\mathrm{K}^{\prime}$ dimi- nuant mais restant très grand, la perte de charge augmente et $\lambda$ varie d'abord proportionnellement à $1 / K^{\prime 2}$ tant que les aspérités sont très espacées les unes des antres, $K^{\prime}$ diminuant encore, les aspérités se resserrent et réagissent les unes sur les autres, la croissance de $\lambda$ est moins rapide. Pour une certaine valeur de $h^{\prime}$, $\lambda$ sera maximum, puis les aspérilés se rapprochant se trouvent dans le sillage les unes des autres of $>$ décroît jusqu'à ce que toutes les aspérités soicnt au contact les unes des antres, disposition qui donne la limite pratique de $\%$ si on suppose que les aspérités peuvent s'imbriquer les unes dans les autres, leur hauteur efficace diminue et finalement pour $\mathrm{K}^{\prime}=0$ on retombe sur le cas d'une paroi lisse. On remaique, en particulier, que pour une valeur domnée du nombre de ReyNoLds, il y a 2 valeurs de $K^{\prime}$ qui donnent le même $\lambda$. La valeur de $K^{\prime}$ qui correspond à la disposition de Nukeradse se trouve sur la branche comprise entre le maximum et $\mathrm{II}^{\prime}=0$; il existe donc une deuxième valeur de ${ }^{\prime \prime}$ qui alonne la même perte de charge que la disposition de NikuRadse.

Nous avons vu plus natul que dans te domaine de l'écoulement turbulent rugueux, la loi de répartition des vilesses suivant un diametre de la section peut s'écrire $a / v_{*}=\mathrm{A}+\mathrm{B} \log y / \mathrm{K}, \mathrm{A}$ el $B$ étant des constantes que Nucuradse a déterminé pour son lype particulier de rugosité et trouvé respectivement égales à 8.48 et 5.75 . Les essais de Schlichting (Experimentelle Untersìchùngen zùm Raùhigkeitsproblem Ing. Arch. Fév. 1936) nous ont permis de calculer la valeur de $A$ pour les aspérités éludiées en prenant pour If la hauteur de l'aspérite. Les valeurs ohtenues diffèrent généralement de celle de Nikukause. Les valeur's de $A>8,48$ correspondent à des rugosités qui se situent entre la rugosité NuKunadse et le parfaitement lisse, les valeurs de $A<8,48$ correspondent au contraire à des rugosités plus fortes que celle de Nikuradse.

Portons les valeurs de $K$ ainsi calculées dans un diagramme, avec $\mathrm{A}$ en ordonnee el $\log \mathrm{V}_{*} \mathrm{~K} / \mathrm{v}$ en abscisse. Dans un tel diagramme, tous les points relatifs à une rugosité type Nuinadese se situent sur une mème horizontale d'ordonnée 8,48. Si nous portons maintenant sur ce diagramme les différents points relatifs à une mème aspérité ( $K$ constant), mais à des densitís différentes ( $K^{\prime}$ variable), on constate que ces points s'alignent sur une même droite. Si on fait choix d'une hauteur d'aspérité différente, ou d'un autre type d'aspérité, on obtiondra encore, en fonction de la densité des poinls qui s'aligneront sur une droite différente de la précédente, mais présentant la même pente, soit :

$$
\mathrm{A}=a-31 \log \frac{\mathrm{V}_{*} \mathrm{~K}}{\mathrm{v}}
$$


a étant une constante indépendante de la densite. mais variant arec la forme of la hauteur de l'aspeirité.

Pour un type d'asperite domné (par exemple la forme sphere ou la forme grain de sable), à chaque point du diagramme correspond une rurugosité caractériséc par une hauteur d'aspérité $K$ et une valeur de la densité. On peut éga-

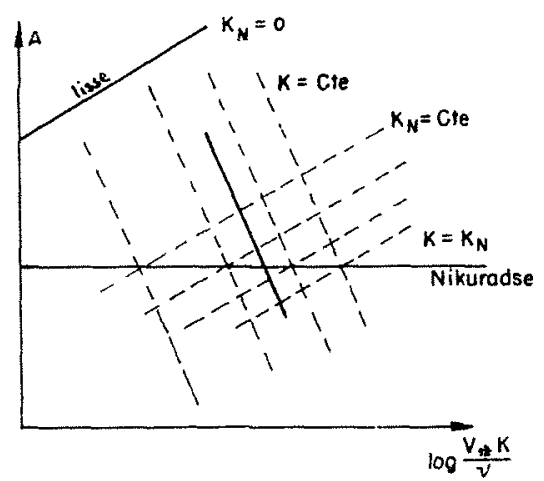

Fici. 6

lement caractériser chaque rugosité, c'est-a-dirc chaque point du diagramme, par la hauteur d'aspérité $K_{N}$ de la rugosité Nikcradse qui donne lá même perte de charge. Il sera ainsi possible de lracer sur le diagramme un réseau comporlant des droites à $\mathrm{K}=\mathrm{C}^{\mathrm{t} \prime \prime}$ et des droites à $\mathbf{K}_{\mathrm{N}}=\mathbf{C}^{\text {te }}$ ou $\mathbf{a} \mathbf{K}^{\prime}=\mathbf{C}^{* *}$. Sur le diagramme représenté, on a tracé le réseau des droites à $K=C^{t}$ et $K_{X}=C^{t e}$ pour le type d'aspérité « sable Nururadsi: ». Nalurellement, la droite $K=K_{N}$ est l'horizontale d'ordonnée 8,48 qui correspond à la densite Nikeradse. Les droites $K_{x}=C^{t *}$ sont toutes parallèles à la droite du turbulent lisse qui correspond à la valeur $K_{X}=0$. Ces résultats ont été obtenus pour le domaine relativement restreinl que les essais de ScrurchTrNG ont permis d'explorer; il serait inéressant de roir dans quelle mesure il est possible d'extrapoler. Pour un autre type de rugosité que le grain de sable, l'horizontale Nikurabse s'obticndra pour un rapport $K / K_{y}$ différent de 1 of caractérisant la hauteur efficace des asperitis en prenant l'aspérité Nikuriusz pour étalon.

I a valeur de d donne une idé assez exacte de l'importance de la perle de charge. Si, dans le diagramme précedent, on fait une coupe le long d'une droite $A=a \ldots 31 \log V_{*} K / v$, on obtient une représentation assez analogue à celle que nous avions indiqué pour les rariations du coefficient $\lambda$ à $\mathbf{R}_{e}=C^{\text {te }}$ en fonction de la densiti des aspérités. Pour une aspérité donnéc, partanl de A lisse pour la densité 0 , la valeur de A diminuc quand la densiti augmente, atteint un minjmum généralement plus petit que 8,48 , puis croit a nouveau lorsque la densite devient infinic.

\section{ENCOMBREMENT DES ASPERTTES}

La présence d'aspérités sur la paroi d'une conduite, surtout si ces aspérités sont importantes, pose un problème pour le choix du diamètre de la section el de son rayon hydraulique. Or, remarquons que dans la formule fondamentale de la perte de charge :

$$
\frac{\Delta h}{\Delta l}=\frac{\lambda}{D}-\frac{u^{2}}{2 g}
$$

le diametre intervient en fait avee la puissance j. En effet. la vitesse moyemue " n'étanl pas directement mesurable, on fait apparaitre $u=\frac{Q}{\pi D^{2} / 4}$. Dès lors, une petite erreur sur la valeur de D entraine une erreur cing fois plus grande sur la valeur de $\triangle h$. L'encombrement des rugosités ne saurait ètre néglige dis que les aspérités sont grandes, pour fixer les idces, a partir de $\mathrm{K} / \mathrm{R}=0,05$. Il laul voir là l'unc des raisons pour lesquelles il est difficile d'extrapoler aux grosses rugosites relatives les résultals de mesures taites sur de labbles rugosiles relatives. C'est ainsi que la formule classique de Nóntodse:

$$
\lambda=\frac{1}{(2 \log \mathrm{R} / \mathrm{K}+1,74)^{2}}
$$

ne doit pas être eonsidéréc comme valable pour les fortes rugosités relatives. Pour les fortes pertes de charge, celle formule conduit, en effel, at des hauteurs d'aspérités exagérés. Pour $R / K==1$, la section est completement encombrée par les aspérités; or, pour ce cas extrème, on obtient seulement $\lambda=0,33$, ce qui est absurde. Cet exemplo est bien propre a montrer a quel point il faut so méfier de l'extrapolation de relations dont la forme mathématiqne ne fat que cachor le caraclère empirique.

\section{ECOTLEMENT DANS RA ZONE DE TRANSITION}

1" Cas d'une rugositi NikvRadse. - On a vu que les régimes exlrêmes du turbulent lisse et du turbulent rugueux itaient caracterises respectivement par les lois de perte de charge suivantes :

$\frac{1}{\sqrt{\lambda}}=2 \log \frac{R_{c} \sqrt{\lambda}}{2,51} \quad$ et $\quad \frac{1}{\sqrt{\lambda}}=2 \log \frac{3.7 \mathrm{I}}{\mathrm{K}}$

Posons plus généralement :

$$
\frac{1}{\sqrt{\lambda}}=2 \log \left\lceil\because \frac{\mathrm{R}_{r} \sqrt{i}}{2.51}+\beta \frac{3.7 \mathrm{D}}{\mathrm{K}}\right\rceil
$$

$x$ et $\beta$ étant deux fonctions de $\frac{V_{*} K}{v}$. 
Colnbrook a supposé que dans la zone de transition on pouvait prendre $x=\beta=1$, ce qui n'est guère admissible que pour une bande assez limitée de valeurs de $\mathrm{R}_{c}$. Cherchons plutòt quelle peut ètre l'allure de la variation des fonctions de $x$ et $\beta$. Pour cela, nous nous appuicrons sur les résultats de Niktradse d'une part, et, d'autre part, nous nous fixerons une relation entre $\alpha$ et $\beta$. soit $\alpha=1-\beta$.

On obtient ainsi, pour la variation de $x$ et de $\beta$ en fonction de $V_{*} K / y$, des courbes avant l'allure

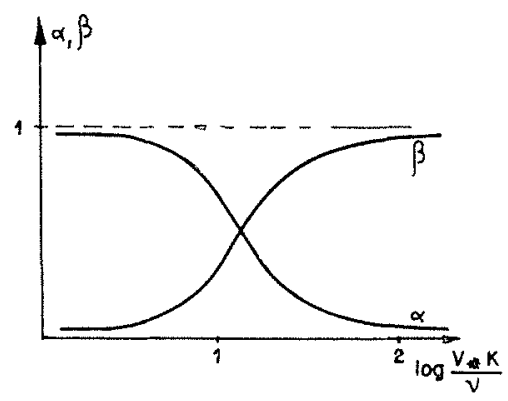

Fig. 7

indiquée par la figure rappelant assez fidèlement les variations d'une tangente hyperbolique. Nous basant sur cette ressemblance toute physique, posons :

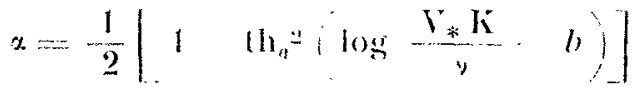

$$
\begin{aligned}
& \text { el } \beta=\frac{1}{2} \mid 1+\operatorname{th}_{c}=\left(\log \frac{V_{*} K}{y} \ldots b\right)
\end{aligned}
$$

En remarquant que $V_{*}=\frac{\sqrt{\lambda} u}{\sqrt{8}}$, on obliendra, en reportant $\%$ et $\beta$ dans lexpression de $1 / \sqrt{\pi}$ :

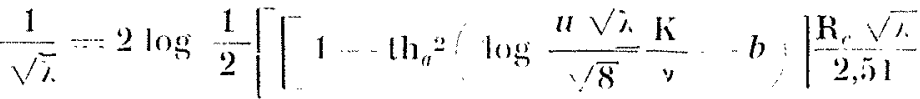

$$
\begin{aligned}
& \left.+\left[1+\mathrm{h}_{n}{ }^{2} \log \frac{u \sqrt{\lambda}}{\sqrt{8}} \frac{\mathrm{K}}{y}-\cdots \mathrm{b}\right] \frac{3.7 \mathrm{D}}{\mathrm{K}}\right]
\end{aligned}
$$

La base a de la langente hyperbolique et la constante $b$ sont choisies pour serrer de pres les résultats de Nowradse; on trouve ainsi $a=3,2$ et $b=1,115$. Toutes réductions faites, on obtient l'expression générale de $\lambda$ pour la rugosité NikrR.ADSF :

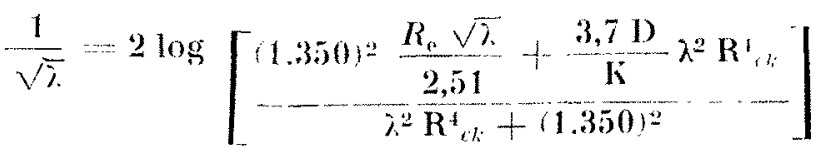

avec :

$$
\mathbf{R}_{e}=\frac{u \mathbf{D}}{\nu} \quad \text { a } \quad \mathbf{R}_{e k}=\frac{u \mathbf{K}}{\nu}
$$

Cette expression fournit les valeurs de $\lambda$ en bonne concordance avec les résultats de NucRADSE. Voici, par exemple, les résultats obtenus pour une rugosite relative $K / R=1 / 126$ et pour différents régimes :

Turbulent lisse :

$R_{e}=10 . \pm 00$ NILIR.ADse $\lambda=0,031$ Calcul $\lambda=0,0304$

Transilion :

$\mathrm{R}_{r}=26.700$ NikLmadse $\lambda=0,026$ Caleul $\lambda=0,0248$

Turbulent rugueux :

$R_{c}=146.000$ Nikr Radse $\lambda=0,027$ Calcul $\lambda=0,0267$

2" Cas d'une rugosite de densilé moiable smr fond parfaitement lisse. Dans un lel cas, on sait quaux grands nombres de Rexwolos une partie de la paroi se comporte comme lisse ef l'autre comme rugueuse, en sorte que les fonctions $\alpha$ et $\beta$ ne varient pas entre 0 el 1 commo dans le cas précédent. mais respectivement entre 1 et 1 - - d'une part, et entre 0 et o d'autre part, s etant une quantité fonction de la densité des ispérités. On posera alors :

$$
\begin{aligned}
& \because \cdots 1-?_{2}\left|1+\operatorname{lh}_{u}{ }^{2}: \log \frac{V K}{y}-b\right|
\end{aligned}
$$

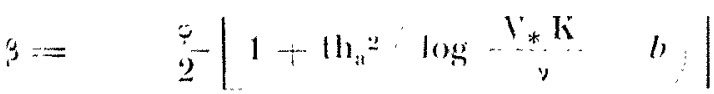

La quantité e est nulle lorsque la densile est nulle; elle croil d'alurd propertionnellement a lat densité, atteint la raleur maximum 1 pour une

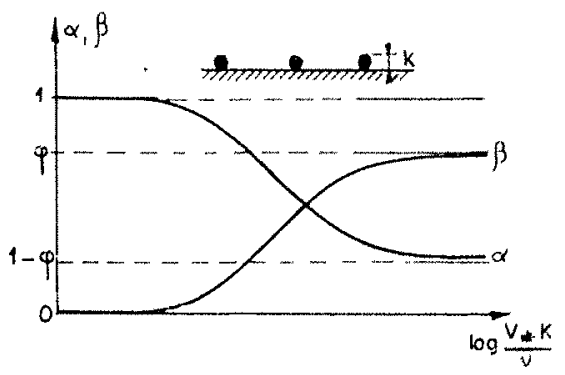

Fit: 8

densilé voisine de celle de Nounanss, puis diaroit et tend vers 0 pour une densité infinie. En portant ces nouvelles valeurs de $\%$ el $p$ dans 
rexpression générale en $1 / \sqrt{\lambda}$, on obtiendra la relation suivante :

$$
\begin{aligned}
\frac{1}{\sqrt{\lambda}}=2 \log [\varphi & \frac{\lambda^{2} \mathrm{R}_{e k}^{4}}{\lambda^{2} \mathrm{R}_{e k}^{+}+(1.350)^{2}} \\
& \left.\left(\frac{3,7 \mathrm{D}}{\mathrm{K}}-\frac{\mathrm{R}_{e} \sqrt{\lambda}}{2,51}\right)+\frac{\mathrm{R}_{c} \sqrt{\lambda}}{2,51}\right]
\end{aligned}
$$

dans laquelle $\mathbf{R}_{e}$ et $\mathbf{R}_{e k}$ ont les mêmes valeurs que précédemment. Pour les grands nombres de RExiolds, cette expression tend vers la relation plus simple :

$$
\frac{1}{\sqrt{\lambda}}=2 \log \left[(1-\psi) \frac{\mathrm{R}_{e} \sqrt{\lambda}}{2,51}+\varphi \frac{3,7 \mathrm{D}}{\mathrm{K}}\right]
$$

La fonction $\varphi$, qui dépend de la densité des aspérités, doit être déterminée expérimentalement pour chaque type d'aspérité.

Il serait possible, d'une manière analogue, de traiter le cas d'une rugosité constituée par de grosses aspérités de densité variable sur un fond rugueux comportant des aspérités de plus petite dimension. L'expression générale de $\lambda$ que l'on obtiendrait dans ce cas serait assez compliquée. Pour les grands nombres de ReynoldDs, cette expression devient :

$$
\frac{1}{\sqrt{\lambda}}=2 \log \left[\beta_{1} \frac{3,7 \mathrm{D}}{\mathrm{K}_{1}}+\beta_{2} \frac{3,7 \mathrm{D}}{\mathrm{K}_{2}}\right]
$$

$K_{1}$ et $K_{2}$ étant respectivement les hauteurs des grandes et petites aspérités $\beta_{1}$ et $\beta_{2}$ des quantités fonction respectivement de la densité de chacun des types d'aspérités. Si les grandes et petites aspérités sont d'un même type, avec une même densité relative, on a :

$$
\beta_{1}+\beta_{2}=1 \text { et } \frac{\beta_{1}}{\mathbf{K}_{1}}+\frac{\beta_{2}}{\mathbf{K}_{2}}=\frac{1}{\mathbf{K}_{e}}
$$

$\mathrm{K}_{c}$ étant la hauteur d'aspérité unique équivalente à l'ensemble.

Dans la pralique industrielle, le type de rugosité Nikcradse se rencontre assez rarement. En particulier, la plupart des mesures industrielles portant sur des conduites pour la zone de transition donne une courbe de variation de $\lambda$ en fonction de $\mathrm{R}_{e}$ qui ne présente pas l'inflexion caractéristique des courbes de Nikunadse. Ceci est dû au fait que la rugosité la plus fréquem- ment rencontrée peut se représenter schématiquement par de grosses aspérités relativement espacées sur un fond lisse au rugueux. ColeBRook, qui est parti d'un tel schéma, obtient effectivement dans la zone de transition une expression de $\lambda$ qui cadre bien avec les faits; malheureusement, son expression n'est valable que pour la zone de transition.

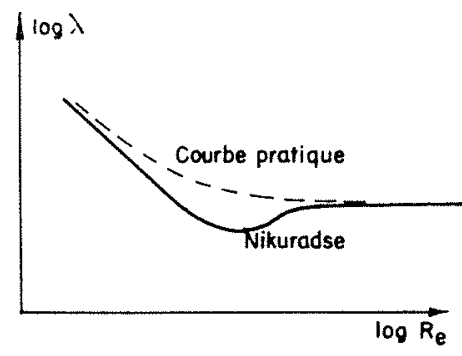

Fig. 9

Les expressions que nous avons indiquées plus haut sont évidemment d'un maniement un peu lourd. Nous avons cherché à les simplifier, en faisant apparaître non pas directement la hauteur des aspérités de la paroi et leur fonction de densité, ce qui n'est pas commode, mais la hauteur d'aspérité d'une rugosité Nikuradss: équivalente aux très grands nombres de Rervolds (domaine quadratique). Il suffit, pour déterminer cette rugosité équivalente, d'une seule mesure de perte de charge faite à une valeur élevée du nombre de Rexvolds. Le coefficient de perte de charge est alors donné par l'expression suivante :

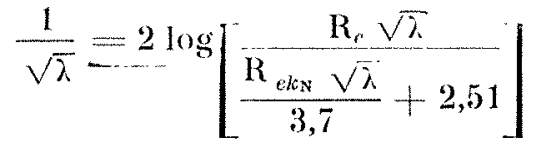

Dans cette expression, $\mathrm{R}_{c}$ a la valeur habituelle et $\mathrm{R}_{\text {er: } \mathrm{N}}$ est le nombre de Reynolds relatif à la hauteur $K_{\mathrm{N}}$ de l'aspérité type Nukuradse qui, dans le domaine du turbulent rugueux, donne la même perte de charge que la conduite considérée. Cette formule a l'avantage d'ètre valable pour tout le domaine de l'écoulement turbulent, du lisse parfait au rugueux parfait et de donner, dans la zone de transition, une loi de variation de $\lambda$ qui concorde avec la plupart des essais industriels. 


\section{III. - INFLUENCE DE LA FORME DE LA SECTION}

\section{IAS SINGILARITÉS DE RAYON HYDRALLIQTE}

Nous avons ru, an début de cet exposé, que si on admet que la force langentielle exercée par le fluide sur la paroi a la même valeur en chacpue point de celle-ci, on voit apparaitre dans l'expression de la perte de charge le rapport de la section de la veine au périmètre de cette section. Ce rapport, appelé rayon hydraulique, suffit à caractériser la section si l'hypothèse de la constance de la force tangentielle $\tau$ est bien vérifiée. Ce sera le cas naturellement pour une section circulaire. On a l'habitude de traiter les sections non circulaires comme des sections circulaires, en introduisant simplement, dans l'expression de la perte de charge, la valeur du rayon hydraulique qui correspond à la section considérée. Cette façon de procéder a besoin d'être justifiée. On sait, en effet, parfaitement que, mis à part le cas de la section circulaire, la force tangentielle à la paroi n'est généralement pas constante. Des mesures de répartition de vitesse permettent de déterminer le gradient de vitesse à la paroi et par suite la force tangentielle. Les résultats de ces mesures appliqués à différentes sections carrées, rectangulaires, triangulaires, etc., mettent toujours en évidence des variations importantes de $\tau$ le long des parois. Mais, chose curieuse, en dépit de ces variations de $\tau$, on constate que tout se passe comme si l'hypothèse de la constance de $\tau$ était vériftée. Il existe donc bien des variations locales de la valeur de $\tau$, mais la valeur moyenne pour tout le périmètre de la section est telle que, en général, la perte de charge calculce brutalement en assimilant la section considérée à une section circulaire de même rayon hydraulique donne un résultat correct. Ceci, toutefois, n'est valable que pour des sections pas trop anormales, comme le carré, le triangle, etc., mais ne s'appliqucrait pas du tout à une section anormale dans le genre de celle figurée ci-dessus. En effet, pour une telle section, l'expérience montre que tout se passe comme si seule la section circulaire intervenait; par contre, lapplication brutale de

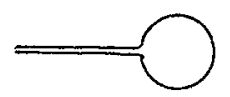

FIG. 10

la règle du rayon hydraulique conduirait à une perte de charge exagérée par suite du développement excessif du périmètre. Quand done dirons-nous qu'une section est normale ou anormale? Affaire de bon sens? Dans tous les cas, toute section qui peut se décomposer aisément en éléments différents, comme l'exemple indiqué ci-dessus, doit être effectivement décomposé et non pas tritité d'un seul bloc.

Avant de poursuivre dans cette voie, il nous faut d'abord examiner un aspeet particulier de la notion de rayon hydraulique. Jusqu'iei, le rayon hydraulique tel que nous lavons defini n'est qu'une caractéristique géométrique de la section considérée.

Un écoulement avec perte de charge n'est pas autre chose qu'un phénomène de transformation et de dissipation d'énergie. L'écoulement ayant lieu en régime uniforme, lénergie potentielle de l'eau (pesanteur, pression) est transformée à la paroi en inergic cinélique de lurbulence, cette dernière inergie diffusée dans toute la masse se transforme ensuite en chaleur par frottement visqueux. Considirons un tronçon de conduite de section $\omega$, de perimetre $P$ et de longueur $\Delta l$. Soit $E_{1}$ lénergie produile a la paroi dans ce troncon et Eo l'energic dissipie en chaleur dans la masse. Si ${ }^{2}$ est l'únergie produite par unite de surface de la paroi, $\mathrm{E}_{1}=e_{1}$ P.Jl, de mème si o.. esl l'énergie dissipee en chaleur par unite de volume, on aura $\mathrm{E}_{2}=e_{2} \omega \Delta l$. Dès lors, comme $\mathrm{E}_{1}=\mathrm{E}_{2}$, on voit immédiatement que :

$$
c_{1} \mathrm{P}=e_{2} \omega=\rho_{2} \mathrm{P} \cdot \mathrm{Rh}
$$

c'est-à-dire :

$$
\mathrm{R}_{h}=\frac{t_{1}}{r_{2}}
$$

le rayon hydraulique apparail alors comme je rapport de l'énergie produite par unité de surface de paroi à l'énergie dissipé par uniti de volmme de la veine fluide. Ceci en admellant, naturelleasent, que la production d'énergie est uniforme sur toute la paroi ef que la dissipation est uniforme dans toute lat veine, ce qui nous ramene a l'hypothese initiale de l'uniformité de: l'effort langenticl a la paroi. Mais si on possede un moyen pour déterminer le volume dans lequel se dissipe l'énergie produite sur un élément donné de la paroi, il sera possible d'élendre les considérations précédentes au cas ou l'efrort tangentiel ne sera pas uniformóment réparti a la paroi. Evidemment, il nous faut, pour cela, introduire d'autres éléments que les ćlements géométriques définissant la section. Supposons connue la répartition des vitesses dans la section sous la forme de l'ensemble des courbes isolaches. Si la turbulence est isotrope, le flux a travers les courbes orthogonales aux isotaches est nul et l'énergie dissipée dans la zone comprise 
entre la paroi ef deux orthogonales lelles que $\mathrm{AO}$ et $\mathrm{BO}$ sera égale a l'énergie produite sur l'élément de paroi $A B$. On a done le moyen de

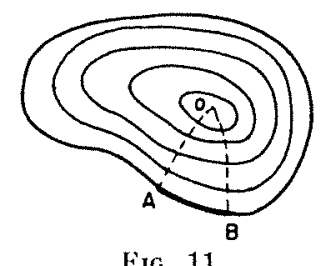

Fif. 11

délimiter la zone de la veine qui correspond à chaque élément de la paroi.

\section{LES SECTIONS DE FORME COMPLEXE}

Revenons maintenant au problème de la division d'une section de forme complexe en sections élémentaires. D'après ce que nous venons de dire, si la répartition des vitesses est connue, le problème est immédiatement résolu, malheureusement, ce ne sera généralement pas le cas. Il faudra donc adopter une division approchée, en se guidant, par exemple, sur les normales à la paroi et sur les bissectrices des angles que forment entre eux les segments voisins constituanl le périmètre de la section. Ia perte de charge pour une section élémentaire sera :

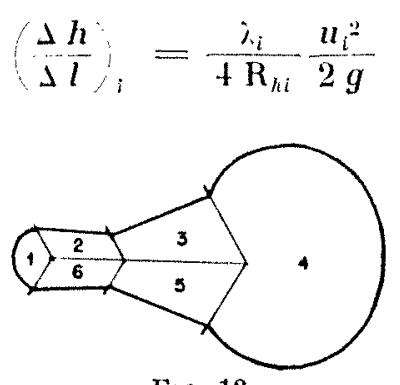

FIG. 12

chaque section élémentaire étant caractérisée par un coefficient de perte de charge $\lambda_{i}$, une vitesse moyenne $u_{i}$ et un rayon hydraulique $\mathrm{R}_{h i}$. On a ainsi autant d'expression de la perte de charge que de sections élémentaires. Ces différentes expressions sont liées par les relations qui expriment que la perte de charge est la même pour toutes les sections élémentaires, soit :

$\frac{\Delta h}{\Delta l}=\frac{\Delta h}{\Delta l}=\ldots . \frac{\Delta h}{\Delta l}=\ldots=\frac{\Delta h}{\Delta l}$

relations auxquelles il faut joindre l'expression de déhil total :

$$
\mathrm{Q}=\omega_{1} u_{1}+\omega_{2} u_{2}+\ldots+\omega_{i} u_{i}+\cdots
$$

Si on se donne les valeurs de $\mathrm{R} h_{i}, \omega_{i}$ (déduite du mode de division de la section en sections bémenlaires) de $\lambda$ qui sera le mème pour toutes les sections, si la rugosité est la même, el de $Q$ on aura la perte de charge, ou au contraire, on déterminera le débit si on connaît la perte de charge. Ce mode de détermination de la perte de charge pour une section de forme quelconque est évidemment criticable par suite de la façon arbitraire dont sont déterminées les sections élémentaires et par suite des hypothèses faites sur la répartition dans la veine du taux de dissipation d'énergie par unité du volume du fluide, mais nous pensons qu'elle est applicable dans de bonnes conditions s'il n'y a pas d'ambiguïté sur la détermination des sections élémentaires.

\section{Cas ou la parol componte plusieurs types} DE RUGOSITÉ

Nous venons d'examiner le problème posé par l'emploi de sections non circulaires pour lesquelles la notion de rayon hydraulique global s'avère insuffisante. Envisageons maintenant le problème posé par une conduite que nous supposons à section circulaire pour simplifier, mais dont le périmètre comporte des zones de rugosilé différentes. Un tel probleme se pose assez souvent dans la pratique avec deux types de rugosile, par exemple une galerie dans le rocher dont seul le radier est revêtu d'un enduit lisse. On peut penser tout d'abord à utiliser la méthode que nous venons d'indiquer par la division de la section en sections élémentaires el cette méthode serait tout indiquée si on connaissail la réparlition des vitesses dans la section. Cetle répartition n'étant pas connue, on effectuera la division en sections élémentaires d'après une répartition de vitesse donnéc à priori. A ce sujet, il faut noter que dans un canal dont la paroi comprend plusieurs types de rugosité, la répartition des vitesses s'organise en sorte que les vitesses diminuent dans le voisinage des regions les plus rugueuses et augmentent dans le voisinage des régions les plus lisses, ceci alin d'assurer l'égalilí des pertes de charge pour les différents canaux élémentaires constituant la veine. Tenant compte de cette remarque, on pourra établir une division en sections élémentaires aussi vraisemblable que possible. Puis on opérera comme il a été indiqué précédemment. Dans le cas de deux rugosités, on aura par exemple :

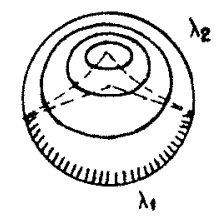

Fig. 13 


$$
\underset{j l}{د h}=\frac{\lambda_{1} u_{1}^{2}}{8 g \mathbf{R}_{h 1}}=\frac{\lambda_{2} u_{2}^{2}}{8 g \mathbf{R}_{h 2}}
$$

avec :

$$
Q=\omega_{1} u_{1}+\omega_{2} u_{2}
$$

Le déplacement des grandes vitesses vers les parois les plus lisses se traduit par une augmentation d'étenduc de la zone dans laquelle se dissipe l'énergie produite par les parois les plus rugueuses. Il se produit donc une diffusion de l'énergie à dissiper dans toute la veine, en sorte que le taux de dissipation d'énergie par unité de volume a tendance à s'uniformiser. Si le taux de dissipation d'énergie a la mème valeur en tout point de la section, on peut admettre que la diffusion de l'énergie à dissiper s'effectue de telle façon que l'on peut appliquer, pour la superposition des énergies provenant de chaque élciment de la paroi, une règle analogue à la loi de DALTON pour l'addition des pressions partielles des différents constituants d'un gaz. Dans un cas, c'est la diffusion par turbulence qui intervient, dans l'autre, c'est la diffusion moléculaire.

On considère donc que l'énergie produite par l'élément de paroi rugueuse est diffusée dans toute la veine, et qu'il en est de même pour l'énergie produite par l'élément de paroi lisse. La perte de charge est alors la somme des pertes de charge partielles correspondant a chaque eliment de paroi, chaque perte de charge partielle śtant calculée avec un rayon hydraulique égal au rapport de la section tolale w à l'élément de périmètre considéré $p_{i}$, d'où, au total:

$$
\frac{\Delta h}{د l}=\frac{n^{2}}{8 g \cdot \omega}\left(\lambda_{1} p_{1}+\lambda_{2}+\cdots+\lambda_{i} p_{i}+\cdots\right)
$$

\section{LAS CANAUX I SURFACE LIBRE}

Un cas limite de conduite comprenant plusieurs types de rugosité est le canal à surface libre. Génćralement ee type de conduite à surface libre est traité par la formule habiluclk

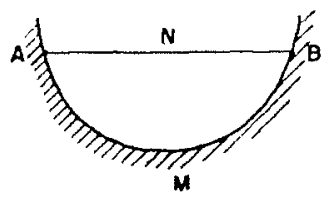

Fig. 14

de perte de charge faisant intervenir le rayon hydraulique, celui-ci étant calculé comme rapport de la section totale de la veine à la longueur du périmètre correspondant uniquement aux parois, soit AMB. La portion de périmètre constituée par la surface libre est négligée, ce qui revient à dire que l'on néglige tout effet provenant de la surface libre. Cette façon de procé- der conduit toutefois à de notables cearts avec la réalité.

Un exemple simple va nous montrer en effet que la surface libre joue certainement un rôle. Considérons un canal semi-circulaire tel que la surface libre constitue exactement un diametre du cercle. Le rayon hydraulique, dans ce cas, a exactement la même valeur que pour une conduite circulaire de mème diamètre en charge. Il faudrait donc s'attendre à obtenir la même perte de charge que pour la conduite circulaire en charge de même diamètre, le débit étant simplement moitie. Or, l'expérience montre que généralement à égalité de perte de charge, le débit est plus petit que la moitié du débit de la conduite en charge. D'autre part, si on relève la répartition des vitesses dans la section, on constate que le maximum de vitesse est un peu audessous de la surface libre et non pas exactement sur la surface libre; comme il aurait fallu s'y attendre, si le canal semi-circulaire était exactement la moitié de la conduite circulaire en charge.

Tout se passe donc comme si certaines forces tangentielles intervenaient à la surface libre pour freiner le mouvement. On ne peut donc, dans le calcul du rayon hydraulique, négliger completement la surface libre. Schokltsch indique que, pour le canal à surface libre de paroi circulatre, on se rapproche de la vérité en faisant intervenir dans le périmetre utilisé pour le ealcul du rayon hydraulique, $40 \%$ du segment représentant la surface libre. Reste à expliquer comment la surface libre intervient pour modifier la valeur de la perle de charge. I'explieation la plus couramment admise est celle de l'existence, a la surface, de forces tangentielles dues à l'entraînement de l'air par l'eau. L'existence de ce phénomène d'entrainement n'est pas douteuse; il y a la une action qui se manifeste effectivement pour accroitre la perte de charge de la veine liquide. Mais, d'autre part, eomple tenu de la difference considerable de masse spicifique de l'eau et de l'air, il faut bien reconnaître que l'influence de l'entrainement de l'air est d'un ordre de grandeur nettement plus faible que celui des phénomènes observés. Done, sams rouloir nier l'influence de l'entraînement, il fatut chercher une autre influence.

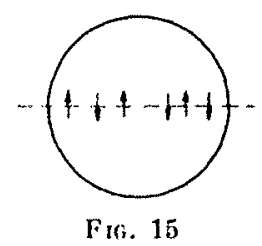

Reprenons l'exemple du canal a surface libe de section semi-circulaire; un tel camal ne peut 
pas etre consideré, en toule rigueur, comme rèsultant de la section par un plan diametral d'une conduite circulaire en charge. En effet, par suite de la turbulence, n'importe quel plan diamćlral de la conduite circulaire est traversé par des paquets de lluide dans un sens ou dans l'autre, constiluant un important échange transversal. Par contre, dans le canal semi-circulaire, aucun échange transversal ne se produit à travers la surface. Dès lors, on peut affirmer que le schéma des échanges turbulents n'est pas le mème dans le canal semi-circulaire à surface libre et dans l'unc des moitiés d'une conduite circulaire en charge. La surface libre joue pour la turbulence un ròle de tranquillisateur, en sorte que les échanges et, par suite, la dissipation d'énergie sont moins actifs dans le voisinage de la surface libre. Nous avons dit plus haul que le rayon hydraulique pourrait être considéré comme le rapport de l'énergie produite par unité de surface de paroi à l'énergie dissipéc par unité de volume de la veine fluide. Par suite de l'effet de tranquillisation de la surface libre, on peut admettre que la dissipation d'énergie s'effectue non pas dans toute la veine, mais dans l'ensenble de la veine, moins une petite région voisine de la surface. Si l'énergie produite à la paroi n'a pas changé, on roit que l'effet de tranquillisation de la surface libre se traduit par une diminution de la valeur du rayon hydraulique, donc une augmentation du cocficient de perte de charge. Schorutrscu, s'appuyant sur l'eflet de l'entraìnement d'air, préconise de calculer le rayon hydraulique comme rapport de la section ali périmètre des parois augmenlé d'une partie du périmètre de surface libre. L'hypothèse de la modification du schéma de turbulence conduit au contraire à calculer le rayon hydraulique comme rapport au périmetre des parois de la section diminuée d'une certaine quantité proportionnelle à l'étendue de la surface libre.

Le probleme de la perte de charge des cananx à surface libre n'est cerles pas cncore completement éclairci; il reste encore un certain nombre de points d'interrogation. Nous voulons parler, entre autres, des curicux résultats obtenus récemment par VARwick. Cet auleur a cherchó a appliquer aux canaux à surface libre les procédés expérimentaux qui araient conduit NikURADSE a ses intéressants résultals sur l’influence de la rugosité. WARWick a donc procédé à des mesures de perte de charge sur des canaux de forme généralement trapézoïdale, dont les parois, comme les conduites de Nikuradse, étaient recouvertes de rugosités artificielles. En jouant sur les profondeur's d'eau et les pentes, il a pu faire varier les nombres de REynolds et les rugosités relatives. Les résultats obtenus sont assez curieux. Pour une dimension donnée des grains constiluanl la rugosite on obtient un faisceau de cour- bes donnant les varialions de $\lambda$ en fonction de $R$, pour dillerentes rugosites relatives. Ce faisceau présente en gros la même allure que le faisceau correspondant des courbes de Nikuradse. Toutefois, ce faisceau se situe nettement au-dessus

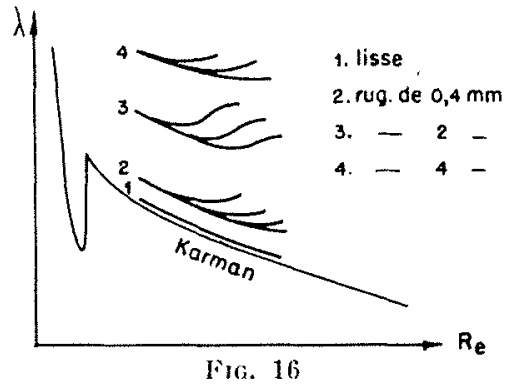

de la courbe de l'écoulement lisse, l'écart étant d'autant plus grand que, pour un mème canal, la grosseur des grains est plus grande. En outre, a égalité de rugosité relative, la transition et le passage au régime rugueux se produisent pour des nombres de RerNolds nettement plus grands en canal qu'en conduite. Il résulte de ces essais que les formules pour conduites ne peuvent être appliquées brutalement aux canaux. En particulier, les formules du type Cnezy à coefficient constant, formules caractéristiques du régime turbulent rugueux, ne s'appliquent pas au domaine prospecté par Varwick; par contre, la formule de BAzIN, qui conduit à une diminution de $\lambda$ lorsque le nombre de RExolos croit, donne de bons résultats. Disons toutefois que la gamme des nombres de Reyouns envisagée nest pas suffisante pour extrapoler ces conclusions. Les singularités mises en évidenee par IVARwick peuvent s'expliquer en partie par les déformations de la surface libre et par l'influence de l'encombrement des rugosités qui conduit à surestimer la valeur du rayon hydraulique (influence non négligeable puisque, en définitive, le rayon hydraulique, ou tout ou moins une dimension caractéristique de la section, intervient à la puissance 5). De plus, les écarts observés s'accordent bien avec l'hypothese de l'effel de tranquillisation de la surface libre que nous avons signalée plus haut. Enfin, peut-être faut-il voir la cause la plus importante des singularités constalées dans lexistence des courants secondaires qui doivent jouer un ròle non négligeable en agissant à grande échelle et d'une facon organisée, comme la turbulence par échange de quantité de mouvements. Notons d'ailleurs que des courants secondaires existent également dans les conduites en charge à section non circulaire, mais leur importance est sans doute moindre que dans les canaux à surface libre. Il est vraisemblable que l'étude de ces courants, dont le qualificatif de secondaire semble diminuer l'inportance, jetterait une lumière nouvelle sur le probleme des pertes de charge dans les canaux. 